\title{
Bayesian statistical approach to binary asteroid orbit determination
}

\author{
Irina D. Kovalenko ${ }^{1,4}$, Radu S. Stoica ${ }^{1,2}$, N. V. Emelyanov ${ }^{1,3}$, \\ A. Doressoundiram ${ }^{4}$ and D. Hestroffer ${ }^{1}$ \\ ${ }^{1}$ IMCCE, Observatoire de Paris, PSL Research University, UPMC univ. Paris 06, univ. Lille1, \\ CNRS, \\ 77 av. Denfert-Rochereau, 75014, Paris, France \\ email: ikovalenko@imcce.fr \\ ${ }^{2}$ Université de Lille, Laboratoire Paul Painlevé, 59655 Villeneuve d'Ascq Cedex, France \\ ${ }^{3}$ M. V. Lomonosov Moscow State Univrsity - Sternberg Astronomical Institute, \\ 13 Universitetskij prospect, 119992 Moscow, Russia \\ ${ }^{4}$ LESIA, Observatoire de Paris, PSL Research University, CNRS, Sorbonne Universités, \\ UPMC Univ. Paris 06, Univ. Paris Diderot, Sorbonne Paris Cité, \\ 5 place Jules Janssen, 92195 Meudon, France
}

\begin{abstract}
The problem of binary asteroids orbit determination is of particular interest, given knowledge of the orbit is the best way to derive the mass of the system. Orbit determination from observed points is a classic problem of celestial mechanics. However, in the case of binary asteroids, particularly with a small number of observations, the solution is not evident to derive. In the case of resolved binaries the problem consists in the determination of the relative orbit from observed relative positions of a secondary asteroid with respect to the primary. In this work, the problem is investigated as a statistical inverse problem. Within this context, we propose a method based on Bayesian modelling together with a global optimisation procedure that is based on the simulated annealing algorithm.
\end{abstract}

Keywords. celestial mechanics, methods: statistical, minor planets, asteroids

\section{Introduction}

The study of binary asteroids may have important implications for the theoretical models of the Solar System's dynamical evolution. For instance, their relative orbit determination allows us to derive the mass of the system, and consequently its density if the size is known: both are essential physical characteristics of the objects.

Parameter estimation directly from observations can be tackled using the inverse problem framework based on Bayesian analysis. This approach is commonly used in astronomy for the determination of unmeasurable parameters from space observations. In the problem of binary orbit determination, these unmeasurable parameters can be the six Keplerian elements: semi-major axis $(a)$, eccentricity $(e)$, inclination $(i)$, longitude of ascending node $(\Omega)$, argument of periapsis $(\omega)$, time of periapsis passage $(\tau)$ with the orbital period $(P)$. These seven parameters are the independent parameters. If the mass of the binary is unknown, the period and the semi-major axis are considered independent, otherwise they are related to each other through the third Keplerian law. The observations consist of a set of time of observation and two coordinates $(x, y)$ on the sky plane of the relative position of the secondary asteroid, with the primary asteroid at the origin.

Several statistical methods for different orbit determination problems have been developed previously. Bayesian orbit computation using the Monte Carlo technique was 
developed by Virtanen et al. (2001). This method, also called statistical ranging, has been investigated for single asteroid heliocentric orbit, described by six parameters, namely the Keplerian orbital elements. The statistical ranging method makes use of the observations sampling within their observational errors and allows to derive the orbital element probability density. A similar approach adding a Markov Chain to parameters' sampling for binaries relative orbit determination was applied by Oszkiewicz et al. (2013). This approach is based on the classical Thiele-Innes method (Aitken 1964) for binary stars, that is combined with a Markov chain Monte Carlo (MCMC) algorithm that uses a Metropolis-Hastings sampler (Hastings 1970). In this problem, which requires seven parameters for orbit determination, sampling was made through observed positions and additional through the orbital period parameter.

In this work unlike previous methods we directly sample orbital parameters, rather than through observations. It allows us on each iteration to avoid computation of orbit from observations, thus avoid solving non-linear equations system (Aitken 1964). Moreover, we combine Bayesian modelling with a global optimization technique in order to derive the best-fit orbit, even with a small number of observations and absence of any initial estimated parameters. To sum up, the method not only allows to examine the orbital element phase space, but also identifies an optimal solution and its margin of error.

\section{Model construction}

Orbit determination means finding the best trajectory parameters fitting their positions to given observations. The observed and calculated positions are related to each other through an observational equation:

$$
D=f(X, t)+\varepsilon
$$

where $D$ is the data given by the set of $N$ observations at times $t=\left(t_{1}, \ldots, t_{N}\right)$,

$$
D=\left(x_{1}, y_{1} ; \ldots ; x_{N}, y_{N}\right) \text {. }
$$

The function $f(X, t)$ computes the sky-plane positions, corresponding to each observation time. The relative motion between observer and binary system as well as time light delay are taken into account. The quantity $X$ represents the set of unknown parameters describing the relative orbit:

$$
X=(a, e, i, \Omega, \omega, \tau, P)
$$

The errors $\varepsilon$ are considered random variables that are supposed to be independently identically distributed. Their distribution is also unknown. Thus, the problem here is given by the determination of $X$ such that the effect of $\varepsilon$ is minimal.

According to this, an a posteriori probability density function is build, which represents the conditional probability density of the unknown parameters $X$ given the observational data $D$. From the Bayes' theorem, this conditional probability density is:

$$
p(X \mid D) \propto p(X) L(D \mid X)
$$

where $p(X)=\exp (-U(X))$ is the a priori density, and $L(D \mid X)=\exp (-U(D \mid X))$ is the likelihood term. The functions $U(X)$ and $U(D \mid X)$ are two functions called, the a priori energy and the conditional data energy, respectively. Their expressions are given in the following. 
Likelihood term The conditional data or the likelihood energy term is defined as the sum of absolute differences between observed and calculated positions.

$$
U(D \mid X)=\sum_{i=1}^{N}\left|D_{i}-f\left(X, t_{i}\right)\right|
$$

The calculated positions are obtained by using within $f$ the current orbital parameters together with the corresponding associate observation time. The residual between observed and calculated positions is

$$
\left|D_{i}-f\left(X, t_{i}\right)\right|=\sqrt{\left(x_{i}^{O}-x_{i}^{C}\right)^{2}+\left(y_{i}^{O}-y_{i}^{C}\right)^{2}}
$$

A priori term The a priori energy term serves as a regularisation term, which constrains the phase space of possible orbits. This term combines density functions of each unknown parameter. For the first approach if there is no a priori information about the parameters, so the distributions are chosen to be uniform on a large range of possible values. For example, $\mathcal{U}[0,1]$ for eccentricity, $\mathcal{U}[0, \pi]$ for inclination and ascending node and $\mathcal{U}[0,2 \pi]$ for the argument of periapsis. The semi-major axis and the orbital period also have uniform a priori distributions, but here their corresponding limits are determined using the observations and the analysis of known binaries in population.

$$
U(X)=\sum_{i=1}^{7} \log p\left(X_{i}\right) .
$$

Proposed solution The estimate of the $X$ vector we propose is the MAP (Maximum A Posteriori), that is the parameter vector value that maximises the conditional distribution Eq. 2.2. This maximum is also the minimum of the sum of the likelihood and a priori energy terms.

$$
\hat{X}=\operatorname{argmax} p(X \mid D)=\operatorname{argmin}[U(D \mid X)+U(X)]
$$

\section{Simulated annealing}

The orbital parameters estimator is computed using the simulated annealing, a global optimisation technique Kirkpatrick et al. (1983). The name of the algorithm is inspired by the annealing processes in metallurgy. In principle, the algorithm converges in distribution to the uniform law on the configuration sub-space made of those values maximising the conditional probability Eq.2.2. This is done by means of an algorithm parameter called, temperature. At high temperatures, all the possible parameter values are taken into account, while at temperatures approaching 0, only those states minimising the energy of the system are accepted. If the initial temperature is high enough, and if the temperature is cooled in the appropriate manner and at a sufficiently slow speed, then the local optima are avoided.

The major steps of the algorithm are:

(a) Fix the initial temperature $T_{0}$ and start a Metropolis-Hastings like algorithm with an initial set of parameters. These initial conditions are given by any value of each unknown parameter in their corresponding definition domain.

$$
X_{0}=\left(a_{0}, e_{0}, i_{0}, \Omega_{0}, \omega_{0}, \tau_{0}, P_{0}\right)
$$

(b) At each iteration $n$ a small change to the current state is done and a new state is obtained: $X_{n e w}$. Practically we change one randomly chosen parameter at a time. This 
Table 1. Astrometric data, where $x=\left(\alpha_{2}-\alpha_{1}\right) \cos \delta_{1}$ and $y=\delta_{2}-\delta_{1}$, where $\alpha$ is right ascension, $\delta$ is declination, refereed to primary (1) and secondary (2), respectively. The $\sigma$ uncertainties in the final digits are indicated in parentheses.

\begin{tabular}{llll} 
UT date & and hour & $\mathrm{x}(\operatorname{arcsec})$ & $\mathrm{y}(\operatorname{arcsec})$ \\
\hline $06 / 08 / 2006$ & 6.3999 & $-0.1718(11)$ & $+0.0630(41)$ \\
$25 / 07 / 2007$ & 1.9308 & $-0.1633(16)$ & $-0.0367(17)$ \\
$07 / 08 / 2007$ & 22.3433 & $-0.2768(21)$ & $-0.0074(10)$ \\
$06 / 10 / 2007$ & 21.5019 & $-0.0528(10)$ & $+0.0455(19)$ \\
$12 / 11 / 2007$ & 9.2313 & $+0.1405(14)$ & $-0.0632(12)$ \\
$25 / 07 / 2008$ & 7.1686 & $+0.1572(13)$ & $-0.0086(14)$ \\
$11 / 12 / 2009$ & 9.4078 & $-0.3653(30)$ & $+0.0379(30)$ \\
\hline
\end{tabular}

change is done within a small neighbourhood of its current state.

Acceptance probability The state $X_{n e w}$ is accepted with the probability:

$$
\alpha=\min \left(1, \frac{p\left(X_{n e w} \mid D\right)^{1 / T_{n}}}{p\left(X_{\text {old }} \mid D\right)^{1 / T_{n}}}\right)
$$

with $T_{n}$ the temperature at the iteration $n$.

Temperature scheduling As stated before, the convergence of the algorithm is reached if the cooling schedule for the temperature is slow enough. Here, the temperature is decreased at the end of each iteration by multiplying it by a constant $0.95<c<1$. Thus the cooling scheme is exponentially decreasing temperature:

$$
T_{n}=T_{0} c^{n}
$$

where $n$ is the iteration number. An alternative cooling schedule that can be considered is $1 /(\log (n)+1)$. In theory, the algorithm should run until the system is completely frozen with a theoretical proved cooling schedule. In practice, a cooling schedule is chosen, then the algorithm starts with a relatively high initial temperature and it runs until a pre-defined low temperature is attained.

\section{Application results}

Here we show an example of a trans-neptunian binary orbit determination. The asteroid is called 2001 UQ18 (Altjira). The following 7 observations (see Table 1) are used (published by Grundy et al.(2011)):

Both time light delay and relative motion between observer and binary were taken into account. For the simulated annealing algorithm we used $T_{0}=1000$ and exponentially decreasing schedule with $c=0.999$. The temperature is decreased every 100 iterations. This strategy allows to approach the equilibrium regime for the Markov chain simulated by the algorithm at fixed temperature. In the same time, this choice allows us to slow even more the cooling schedule, hence all together, improving our results. The simulated annealing was stopped when $T=1$. Figure 1 shows how orbital parameters evaluate and converge to solution during the simulated annealing.

At that stage, $10^{5}$ iterations of the algorithm with fixed temperature $T=1$ were run. This is equivalent with a sampling of the a posteriori distribution using a MetropolisHastings algorithm. Being in a stationary regime, statistical inference can be performed. But before this, in order to reduce correlation effects, the samples were considered every 10th iteration. Hence, our statistical analysis is based on $10^{4}$ orbital parameter samples. The resulting values are presented on the Table 2. Figure 2 shows a fitted orbit and astrometric positions projected onto the sky plane. 

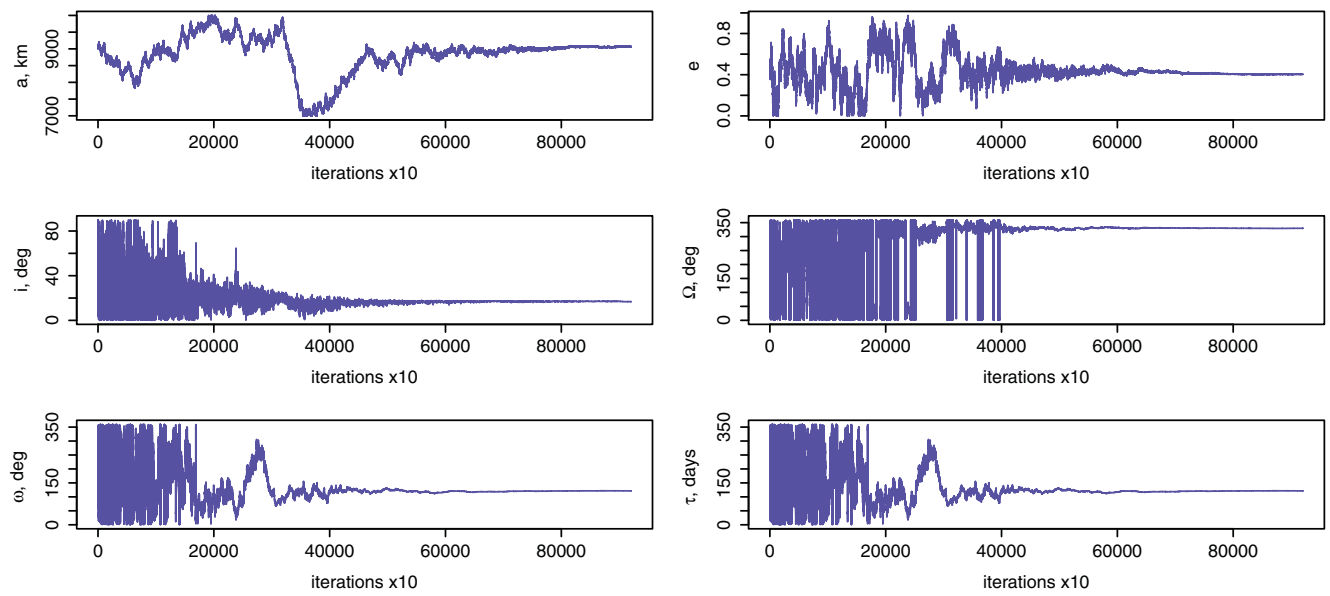

Figure 1. Simulated annealing suite.

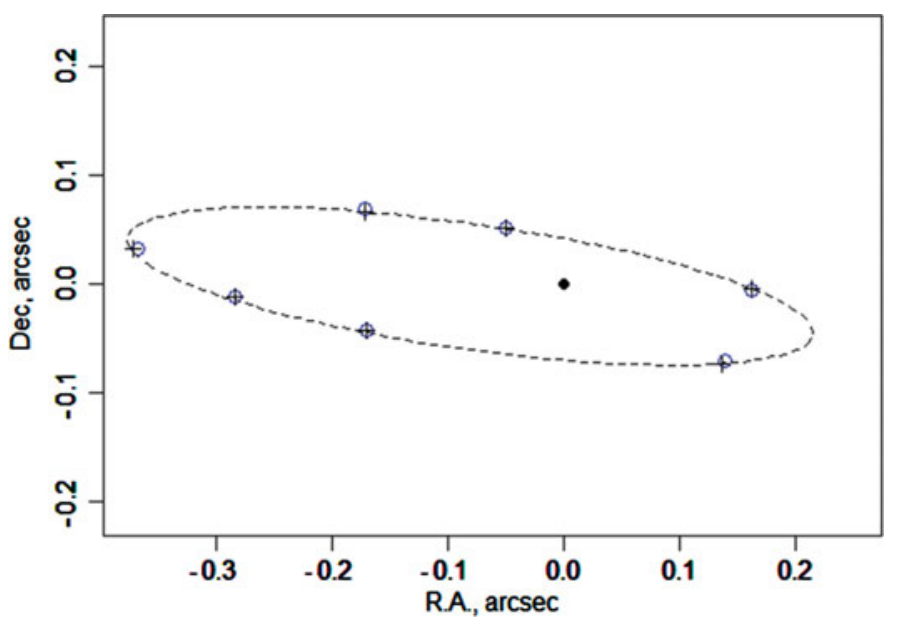

Figure 2. Relative astrometric positions and fitted orbit projected onto the sky plane. Solid circles at the origin is the primary. Open circles show observed relative positions. Plus signs is the mean fit orbit solutions predicted positions at the observation times. Large dotted line represents the sky plane projection of the mean fit orbit at the mean time of the observations.

Table 2. Fitted elements' summary for the asteroid 2001 UQ18.

\begin{tabular}{lllll} 
Parameters & Mean $\pm \sigma$ & 1st quantile & Median & 3rd quantile \\
\hline Semimajor axis, km & $9983.3 \pm 7.35$ & 9978.8 & 9984.0 & 9988.7 \\
Eccentricity & $0.3524 \pm 0.0003$ & 0.3522 & 0.3524 & 0.3526 \\
Inclination, deg & $35.641 \pm 0.025$ & 35.619 & 35.652 & 35.653 \\
Longitude of asc. node, deg & $311.98 \pm 0.018$ & 311.96 & 311.98 & 311.99 \\
Argument of periapsis, deg & $148.51 \pm 0.01$ & 148.50 & 148.51 & 148.51 \\
Time of periapsis, RJD & $54251.81 \pm 0.01$ & 54251.81 & 54251.81 & 54251.82 \\
Period, days & $139.633 \pm 0.009$ & 139.633 & 139.634 & 139.637 \\
\hline
\end{tabular}

\section{Method verification}

The simulated annealing method has been compared with a classical least squares method. The classical method can not be applied to the case of asteroid Altjira because of small number of observations. Moreover this method requires a good parameters 
Table 3. Results for the asteroid Emma.

\begin{tabular}{lll} 
& Simulated annealing & \\
\hline Semimajor axis, $\mathrm{km}$ & $582.753 \pm 1.33$ & $572.074 \pm 19$ \\
Eccentricity & $0.11043 \pm 0.001$ & $0.1378 \pm 0.02$ \\
Inclination, deg & $99.24 \pm 0.79$ & $96.39 \pm 5$ \\
Longitude of asc. node, deg & $342.24 \pm 0.53$ & $342.35 \pm 6.6$ \\
Argument of periapsis, deg & $344.68 \pm 0.15$ & $345.37 \pm 11.5$ \\
Period, days & $3.388 \pm 0.00001$ & $3.365 \pm 0.006$ \\
Mean anomaly $^{b}$, deg & $28.5 \pm 0.18$ & $10.9 \pm 10.4$ \\
\hline
\end{tabular}

(a) The uncertainties are due to the algorithm's configurations. (b) At the epoch 53320.4 MJD.

initialisation. We also tested our method using 22 observations of an asteroid Emma published in Marchis et al. (2008). The least squares method proved to be applicable to this asteroid. Thus the orbital parameters of Emma were obtained by both simulated annealing and least squares methods. The results with estimated uncertainties are shown in the Table 3 .

\section{Conclusions and perspectives}

The proposed method have good results for the asteroid 2001 UQ18 and also for several other known binaries. At the same time, the method showed results comparable to the using classical least square method. Our model and cooling schedule choices were chosen after trail and error. The choice of an optimal cooling schedule for a given problem is an unsolved mathematical problem. Moreover, we can complete the model construction: the a priori as well as the likelihood functions. The study of the parameters variability due to observations uncertainties are also in our future work. We anticipate applying the developed method to new observations from the Gaia satellite, which should allow us to use data with unprecedented accuracy (Pravec \& Scheirich 2012).

\section{Acknowledgements}

This work is supported by Labex ESEP (ANR N 2011-LABX-030).

\section{References}

Aitken, R. G. 1964, New York: Dover Publication, 1964

Grundy, W. M., Noll, K. S., Nimmo, F., et al. 2011, Icarus, 213, 678

Hastings, W. K. 1970,Biometrika, 57(1), 97-109

Kirkpatrick, S., Gelatt, C. D., \& Vecchi, M. P. 1983, Science, 220, 671

Marchis, F., Descamps, P., Berthier, J., et al. 2008, Icarus, 195, 295

Oszkiewicz, D., Hestroffer, D., \& David, P. 2013, EPSC2013,522, 8

Pravec, P. \& Scheirich, P. 2012, Planetary and Space Science, 73, 56

Virtanen, J., Muinonen, K., \& Bowell, E. 2001, Icarus, 154, 412 